\title{
Towards a Pricean foundation for cultural evolutionary theory
} (Hacia una fundamentación priceana de la teoría de la evolución cultural)

\author{
Lorenzo BARAVALLE* \\ University of Lisbon \\ Victor J. LUQUE \\ University of Valencia
}

\begin{abstract}
The Price equation is currently considered one of the fundamental equations - or even the fundamental equation - of evolution. In this article, we explore the role of this equation within cultural evolutionary theory. More specifically, we use it to account for the explanatory power and the theoretical structure of a certain generalised version of dual-inheritance theory. First, we argue that, in spite of not having a definite empirical content, the Price equation offers a suitable formalisation of the processes of cultural evolution, and provides a powerful heuristic device for discovering the actual causes of cultural change and accumulation. Second, we argue that, as a consequence of this, a certain version of the Price equation is the fundamental law of cultural evolutionary theory. In order to support this claim, we sketch the ideal structure of dual-inheritance theory and we stress the unificatory role that the Price equation plays in it.
\end{abstract}

KEYWORDS: cultural evolutionary theory; Price equation; dual-inheritance theory; fundamental laws; metatheoretical structuralism.

RESUMEN: La ecuación de Price es actualmente considerada una de las ecuaciones fundamentales -o incluso la ecuación fundamental - de la evolución. En el presente artículo exploramos el papel de dicha ecuación dentro de la teoría de la evolución cultural. En concreto, usamos la ecuación de Price para analizar la capacidad explicativa y la estructura teórica de una versión general de la teoria de la herencia dual. En primer lugar, argumentamos que a pesar de carecer de un contenido empirico definido, la ecuación de Price ofrece una adecuada formalización de los procesos de evolución cultural y ofrece una poderosa herramienta heuristica para el descubrimiento de las causas concretas del cambio y acumulación cultural. En segundo lugar, argumentamos que, como consecuencia de esto, una cierta versión de la ecuación de Price es la ley fundamental de la teoria de la evolución cultural. Para defender esta afirmación delineamos la estructura ideal de la teoría de la herencia dual y enfatizamos el papel unificador que la ecuación de Price juega en ella.

PALABRAS CLAVE: teoría de la evolución cultural; ecuación de Price; teoria de la herencia dual; leyes fundamentales; estructuralismo metateórico.

* Correspondence to: Lorenzo Baravalle. Universidade de Lisboa, Campo Grande; Edifício C4, $3^{0}$ piso, Sala 4.3.24 (Lisbon, 1749-016; Portugal) - Ibaravalle@fc.ul.pt - https://orcid.org/0000-0001-6299-6403

How to cite: Baravalle, Lorenzo; Luque, Victor J. (2022). «Towards a Pricean foundation for cultural evolutionary theory»; Theoria. An International Journal for Theory, History and Foundations of Science, 37(2), 209-231. (https://doi.org/10.1387/theoria.21940).

Received: 2020-08-07; Final version: 2021-08-09.

ISSN 0495-4548 - eISSN 2171-679X / (c) 2022 UPV/EHU

This work is licensed under a

Creative Commons Attribution-NonCommercial-NoDerivatives 4.0 International License 


\section{Introduction}

Formulated at the beginning of the 1970s by population geneticist George Price, the Price equation was initially received with scepticism by most evolutionary biologists, due to its highly abstract and general character. Yet - as many projects of extension of Darwinism to social evolution started emerging in the following decades - this apparent defect turned out to be one of its major strengths. Nowadays, some authors look at the Price equation as the key for a generalised theory of evolution (Frank, 2017). In this article, we shall argue that the Price equation - or, at least, a version of it - is the fundamental law of cultural evolutionary theory.

In order to support this claim, we shall consider two issues. The first one is genuinely theoretical. Cultural evolutionists have identified, throughout the last decades, a set of evolutionary factors responsible for cultural change. In section 2 , we shall shortly summarise them. The question is: may the Price equation properly represent the causes of cultural evolution? After having introduced in some detail the Price equation in section 3, we shall argue -in section 4- that it is possible to answer affirmatively to this question.

The second issue that we shall consider says about the empirical import of the Price equation. Even accepting that the formal descriptions of cultural evolutionary forces can be accommodated within the Price equation, this does not entail that we can satisfactorily employ the equation for explanatory or predictive goals. This is a special case of a more general concern related to the Price equation, namely, that the equation is no more than a mathematical identity and, thus, its practical usefulness is dubious (van Veelen, 2005). We shall attempt to respond to this concern in two parts.

First, in section 5, we shall concede to the critics that the Price equation alone does not directly convey any causal information. Still, we shall argue that if, in a specific scenario, we are able to identify at least some evolutionary forces, then we can employ the Price equation to provide hypotheses about the presence of other causes of evolution and their characteristics. We shall illustrate this point through three main case studies (MacCallum et al., 2012; Aguilar and Akçay, 2018; Gong et al., 2012).

If we compare the Price equation to other "fundamental laws" in physics and biology, like Newton's second law or the "principle of natural selection" (Brandon, 1982), we can easily appreciate that they all are merely consequence laws (Sober, 1984) - that is, they do not have a causal import - and they are almost empirical vacuous (Díez and Lorenzano, 2013) -i.e., they are little more than identities. Therefore, what the critics consider a defect of the Price equation is, on the contrary, a trait that this equation shares with some of the most important laws in the history of science (Luque, 2017; Luque and Baravalle, 2021). It is what Kuhn (1970) called a "generalisation-sketch" and Moulines (1984) a "guiding-principle": a heuristic principle that provides a working scheme for developing the further details of the theory.

We shall fully characterise the Price equation as the fundamental law of cultural evolutionary theory - by employing some conceptual resources of metatheoretical structuralism (Balzer $e$ al., 1987) - in section 6. In doing so, we shall also tentatively depict the overall structure of cultural evolutionary theory. We think that this is an important task, since if attained - it would be evidence that cultural evolutionary theory is somehow unitarily grounded; a claim that has recently been challenged (e.g., Lewens, 2015). 


\section{A short overview of cultural evolutionary theory}

In accordance with a largely shared view, the core of cultural evolutionary theory is constituted by the models that Cavalli-Sforza and Feldman (1981), Boyd and Richerson (1985) and their collaborators (especially Joseph Henrich, 2001, 2004) built within the so-called dual-inheritance theory. ${ }^{1}$ According to dual-inheritance theory, culture is differential transmission and accumulation of learned information. What kind of information is transmitted and accumulated in human cultures is largely dependent on our hard-wired preferences for certain kinds of behaviour. Genetic evolution provided human beings with characteristic social learning biases that have proven adaptive in relatively stable environments, wherein individual trial-and-error learning is unnecessarily costly. They are the disproportionate imitation/emulation of the most common type (conformism), of the most successful type (model-based or prestige biases), and of the most psychologically attractive, memorable or useful type (content biases). ${ }^{2}$ Conformism and model-based biases are sometimes also called "context-based biases".

Our cognitive preferences contribute to determine the distributions of cultural variants (like artefacts, beliefs, items of knowledge, words, symbols, skills, norms, rituals, etc.). In dual-inheritance theory, cultural variants are usually considered as a class of psychological or behavioural phenotypic traits, whose specificity is, precisely, that they are culturally transmitted. ${ }^{3}$ Since the successful transmission of cultural variants depends on the fact that there are individuals who interact so as to satisfy their cognitive biases, it is possible to say that the distribution of a cultural variant is influenced by its attractiveness (due to content biases) and its previous distribution (due to context-based biases). Moreover, since cultural variants are a class of phenotypic traits, their diffusion may be also conditioned by factors that act in any other natural evolutionary process, like natural selection, drift or migration (among others that we shall discuss in more detail below). Most notably, natural selection acts in cultural evolution by eliminating cultural variants that are too detrimental from a biological point of view (i.e., this is the case of cultural variants that drastically reduce the survival or reproductive chances of those who adopt them). In spite of the latter, dual-inheritance theory predicts a large degree of autonomy for cultural accumulation: provided

1 There are alternative conceptions which dispute the centrality of dual-inheritance theory within cultural evolutionary theory (e.g., cultural epidemiology; Claidière et al., 2014). While our intuition is that the Price equation may play a foundational role in these conceptions as well, we shall not discuss them here. As correctly pointed out by an anonymous reviewer, sometimes the paternity of dual-inheritance theory is attributed to Lumsden and Wilson (2005[1981]). Although we acknowledge that Lumsden and Wilson's work has numerous affinities with Cavalli-Sforza and Feldman's and Boyd and Richerson's, the former has been less influential for the following development of the theory. For this reason, we will not take it into account here.

2 This picture has been somehow complexified by recent research (see, for instance, Kendal et al., 2018). For simplicity, we shall not take into account such developments; yet, we think that they may be easily accommodated to the framework we are going to defend here.

3 Some cultural variants - like artefacts, written languages or symbols - are not, strictly speaking, phenotypic traits, but rather objects carrying information potentially influencing phenotypic traits: they are sometimes conceived as the elements making up culturally constructed ecological niches (Odling-Smee et al., 2003). 
that social learning is usually less costly or more effective than individual trial-and-error, partially maladaptive cultural variants may be maintained within a population, evolve and even subvert "genetically-coded" behaviours (classic examples are the evolution of lactose tolerance and the demographic transition in modern times).

In the attempt to systematise all the possible causes of cultural change, the overall effects of content and context-based preferences on the diffusion of a cultural variant are sometimes conceptualised as processes of "cultural selection" (Durham, 1991; Mesoudi, 2011). Some authors regard this characterisation as misleading (e.g., Claidière et al., 2014; Lewens, 2015). From their point of view, it is incorrect (or, more modestly, unnecessary) to characterise social learning biases as selective factors because they do not primarily act as sorting factors (like natural selection, which "filters" the fittest variants from the less fit), but rather as transmission biases (like meiotic drive, which causes the preferential transmission of certain variants previously to selection). As we shall see in the next sections, the distinction between selective factors and transmission biases is crucial for the formulation of the Price equation and its cultural evolutionary derivations. In spite of this, we believe that the choice of characterising social learning biases as selective processes or, alternatively, as transmission biases is largely conventional and depending on the modellers' interests (as a matter of fact, also meiotic drive can be considered as a selective process, if we analyse this phenomenon at the gametic level). ${ }^{4}$ At any rate, and with the goal of providing a framework allowing a characterisation of cultural change as the result of both selective forces and transmission biases, we suggest the following partition (in line with Frank, 2012): if an evolutionary factor is able to modify the "content" of a cultural variant (i.e., its characteristic features, or the kind of behaviour that is related to it), then it should be classified as a transmission bias; if an evolutionary factor only modifies the distribution of a cultural variant, then it should be taken as a selective - or, more generally, "sorting" - force.

4 On this point, see also Acerbi and Mesoudi (2015) and Charbonneau (2020).

5 In order to a avoid the impression that we are uncritically assuming a controversial point, some further remarks are needed. In the following sections, our discussion on the role of the Price equation in cultural evolution will largely rely on Aguilar and Akçay (2018) and El Mouden et al. (2014). Their models crucially include a notion of "cultural influence" that is potentially problematic. This notion is supposedly defined in terms of the successful transmission of cultural traits. However, as observed by Jonathan Birch (personal communication), if cultural influence depends on whether a descendant is likely to acquire the same variant as the ancestor, then El Mouden et al.'s and Aguilar and Ackçay's models do not really separate selection from transmission biases. We recognise that this is an issue for these models (and, more in general, for those explanatory projects relying on the view that fidelity is a description-independent property of cultural transmission; see Charbonneau and Bourrat, 2021). Yet, we believe that this kind of problem can be conceptually distinguished from the ones that we aim to address in this article. To put it in Lewontin's (1974) terms, a fully satisfactory evolutionary theory must be both dynamically and empirically sufficient. An evolutionary theory is dynamically sufficient when it is able to provide, in principle, a causal characterisation of the factors involved in the evolutionary change. Moreover, it is empirically sufficient if these factors can be measured and distinguished in practice. We may say that, although both aspects are fundamental for the explanatory success of cultural evolutionary theory, we here only deal with dynamical sufficiency, while the above problem is mainly related to empirical sufficiency. 
In accordance with such a criterion, we tentatively put forward the following taxonomy (inspired in Mesoudi, 2011 and Baravalle, 2021) of the possible causes of cultural evolution:

- Selective forces: natural selection, cultural selection due to content and context-based biases. Selective forces increase or decrease the frequency of a cultural variant depending of its genetic fitness value (natural selection), its cultural fitness value (cultural selection due to content biases), and its previous distribution (cultural selection due to context-based biases). (The notion of "cultural fitness" will be elucidated later, in section 4). Cultural selection due to context-based biases can be further sub-classified into $a$. Frequency-dependent cultural selection (cultural selection due to conformist preferences), and $b$. Cultural selection dependent on structural features of the population (cultural selection due to prestige biases).

- Random forces: genetic and cultural drift. While genetic drift has an impact on the distribution of a cultural variant because it stochastically changes the number of potential transmitters of the variant, cultural drift is most properly conceived as the effect of the transmission of cultural variants with equal genetical or cultural fitness (thus selectively neutral). Both selective and random forces may be labelled as "sorting" forces, for reasons that will be spelt out later.

- Transmission biases: guided variation, random mutation, blending transmission. Transmission biases introduce variation in the process of cultural evolution. Cultural variation may be introduced by changing previous features of a cultural variant in a way that it makes it expectedly - but not necessarily - fitter, either genetically or culturally (or both). The processes responsible for this kind of directed variation are sometimes called "decision-making" forces, so as to distinguish them from processes changing features of cultural variants in a "blinder" way -that is, in a way decoupled from the increase of genetic or cultural fitness. Differently from the other transmission biases, blending transmission does not create a new variant nor simply modifies an existing one, but rather merges two (or more) existing variants in a new one.

- Migration: demic migration and cultural migration. While demic migration increments the frequency of a cultural variant because more potential transmitters are physically introduced into the population under study, cultural migration is generally due to the presence of some technology (radio, press, tv, internet etc.) that allows the introduction of new cultural variants from outside the population without any physical displacement of individuals.

\section{The Price equation}

George Price developed, in the early 1970s, an abstract formula to represent evolutionary change (Price, 1970, 1972). Populations are composed by entities, usually organisms, which may vary in some characteristics. When these entities are exposed to the challenges imposed by the environment, some of those characteristics may help them to overcome the environmental restrictions. In addition, depending on the existence of an inheritance system faithful enough, these traits may be transmitted to the next generation. These two 
basic processes - that is, selection and inheritance - are represented by the Price equation in a simple algebraic language, in terms of covariances and expectations, as follows (Frank, 2012):

$$
\bar{w} \Delta \bar{z}=\operatorname{Cov}(w, z)+E(w \Delta Z)
$$

where $\Delta \bar{z}$ is the change in average value of a character $z$ (in this case its mean, but it can also be adapted to represent higher moments such as variance, skew, etc.) over an arbitrary time step (usually one generation), $w$ is the absolute fitness of an individual (calculated as the number of descendants), and $\bar{w}$. is the average fitness. The first term on the right-hand side is the covariance between fitness $w$ and the character $z$, and represents the change in the average value of $z$ due to differential survival and reproduction. Notice that, since this term is just a measure of the statistical association between the character and fitness, it does not necessarily represent a causal connection between them. For this reason, although it is typically interpreted as representing natural selection, it represents the effects of drift as well (Rice, 2004). The second term on the right-hand side is the expected value of the quantity $\Delta z$, which measures how much the offspring deviate from their parents with respect to the character $z$ (i.e., it accounts for the change in the value of the character from the parents to the offspring due to processes involved in reproduction). Thus, the second term represents the fidelity of the transmission of the organisms with respect to the character $z$. If the fidelity of the transmission is not perfect, then some evolutionary forces such as mutation, recombination, selection at a lower level of organization, or other processes, are causing a transmission bias.

It is worth stressing some additional characteristics of the Price equation. The high level of abstractness of the Price equation allows it to model a wide range of entities and relationships among them. This lack of specificity is very useful for researchers. It helps them to think on different mechanisms of reproduction (asexual, sexual, etc.) and different mechanisms of inheritance beyond genetic inheritance, including epigenetic inheritance, or behavioural and symbolic inheritance (Helanterä and Uller, 2010). ${ }^{6}$

Because of the lack of substantive biological assumptions, it is usually stated the Price equation does not involve idealisations (Godfrey-Smith, 2009) or that it is an assumption-free statement about evolution (Walsh and Lynch, 2018). As a matter of fact, although the Price equation actually relies on some minimal assumptions, these are not simplifying assumptions. They are instead intended to establish the basic properties of any evolutionary system.7 These properties can be summarised as follows (Rice, 2004, p. 169):

- Change over time: To be considered as evolutionary, a system requires a population of characters changing over generations. In order to represent generations, we may use any time interval that is appropriate for the system under study.

- Ancestor/Descendant relations: Different generations of an evolving population must be connected by some character. This is the basic requirement for inheritance.

6 The Price equation is even able to handle blending inheritance (Gardner, 2011).

As we shall see in section 4, some additional assumptions (such as the conceptualisation of a population as a closed system) are less fundamental, and therefore can be relaxed in order to incorporate more complex scenarios. 
- Character or phenotype: Any feature of an entity that can be represented with a real number is, potentially, a phenotypic character. Accordingly, very different entities - such as alleles, genotypes, groups of individuals, etc. - can be interpreted as endowed with evolving characters.

These broad assumptions behind the Price equation make it an especially good candidate for representing cultural evolution. ${ }^{8}$ As a matter of fact, these conditions are satisfied by cultural change processes. Cultures are changing systems, which can be profitably conceived as composed by sets of cultural variants - which, as we have seen in section 2, can be conceived as a class of phenotypic traits, or objects carrying information potentially influencing phenotypic traits - transgenerationally transmitted. Yet, the original Price equation does not say anything specific about cultural evolutionary processes. As a matter of fact, it can be arguably applied in many other fields outside population genetics (e.g., Alizon, 2009, Govaert et al., 2016), which is the field in which it was originally formulated. In order to support the claim that the Price equation can properly represents all the causes of cultural evolution (as we have depicted them in section 2), we shall now thus proceed considering two attempts to reformulate the equation so as to make it more explicitly suitable to account for cultural evolutionary dynamics.

\section{Modelling cultural evolution with the Price equation}

In recent years several researchers have used the Price equation in different ways to formalise cultural change. Some researchers have adopted the standard Price equation, redefining and rearranging its parts for specific purposes (Henrich and Boyd, 2002, Okasha, 2007). Other researchers, instead, have developed new forms of the Price equation, adding new assumptions in order to obtain a properly cultural Price equation. In this article, we shall focus on the latter, and especially on El Mouden et al. (2014) and Aguilar and Akçay (2018). ${ }^{9}$ As we shall see in this section, both these works show that the Price equation is able to properly represent all the possible causes of cultural evolution. Still, in our opinion, Aguilar and Akçay's equation is more accurate, for reasons that we shall spell out shortly. Then, in the remaining of this article, we shall preferentially take their equation as the correct formulation of the Price equation in the context of dual-inheritance theory.

In their paper, El Mouden and collaborators start by matching individuals $j$ to a set of $A_{j}$ individuals of the previous generation that have had some cultural influence on them. They thus observe that, while the human genetic system of inheritance determines a fixed number of ancestors for each descendant (a human being always has two direct ancestors), cultural influence allows some individuals to have a variable number of ancestors. Some descendants can have a particular cultural trait that may come from different and multiple ancestors without any genetic relationship. Moreover, while genetic inher-

8 It is worth to notice that George Price himself intended the Price equation to capture a general truth about all forms of selection, not just natural selection (Price, 1995). See Rice $(2008,2020)$ and Luque (2017) for an analysis of further extensions of the Price equation framework.

9 See also Birch (2017, chap. 8) for an additional discussion of cultural evolution and the Price equation. 
itance is "discontinuous" (in the sense that an allele is either transmitted to the descendant or not), cultural influence comes in degree (i.e., a cultural variant can be just partially transmitted).

In order to properly model these differences between genetic inheritance and cultural influence, El Mouden and colleagues assign, to each individual $i$ in $A_{j}$, a weight $\Upsilon_{i j}$ representing the influence that an ancestor has had on a descendant with respect to a particular cultural trait. Hence, by connecting ancestors and descendants by social learning rather than genetic parenthood, they define cultural fitness as "a measure of cultural influence, reflecting both the number of people who learn from an individual, and the degree to which their traits are influenced when they do learn" (El Mouden et al., 2014, p. 233), or $c_{i}=\sum_{j \in D_{i}} \Upsilon_{i j}$, where $D_{i}$ is the set of individuals culturally influenced in the next generation. ${ }^{10}$ This implies that in cultural evolution, where social learning is the main vehicle of transmission, the degree of influence is as important as the number of people influenced. By assuming a constant population size $\left(\sum_{i=1}^{N} \sum_{j \in D_{i}} \Upsilon_{i j}=\sum_{j=1}^{N} \sum_{j \in A_{i}} \Upsilon_{i j}=N\right)$, and after some algebra, El Mouden and colleagues present the following cultural Price equation:

$$
\Delta \bar{z}=\operatorname{Cov}(c, z)+E_{c}(\Delta z)
$$

where $\Delta \bar{z}$ is the change in the average value of a cultural trait $z$. The first term on the righthand side represents how much a cultural trait $z$ and cultural fitness, $c$, covary; and $E_{c}(\Delta z)$ represents the transmission bias in cultural change, weighted by cultural fitness $c$. This new cultural Price equation allows these researchers to give formal expressions to concepts like cultural altruism (as a behavioural trait that reduces the cultural fitness of the actor, whilst increasing the cultural fitness of those with whom the actor interacts), cultural relatedness (as the regression coefficient of the relationship between the cultural values of an actor and those of the people with whom he interacts, for a particular culturally transmitted trait), and to derive a cultural counterpart of the famous Hamilton's rule (El Mouden et al., 2014, p. 234).

Drawing on this work, Aguilar and Akçay (2018) have presented a similar formalism to model cultural change. The difference with the previous cultural Price equation is that Aguilar and Akçay include both genetic and cultural inheritance systems in their formalism. ${ }^{11}$ By doing so, they manage to establish one framework for both inheritance systems, tracking both genetic and cultural fitness in the same formalism. This is important because, according to dual-inheritance theory, cultural traits may be influenced by both genetic and cultural factors (see section 2). As a consequence, both genetic and cultural inheritance systems must be included in the same framework in order to predict and explain the fate of cultural traits. In this sense, Aguilar and Akçay gene-culture Price equation is a generalisation of El Mouden et al.'s cultural Price equation, while the latter can be considered as a special case when genetic components are negligible. Thus, Aguilar and Akçay present the

${ }^{10}$ See footnote 5 for some further remarks over the notion of "cultural influence".

${ }^{11}$ El Mouden and colleagues develop, in the Supporting Information section of their article, several scenarios with cultural and genetic systems, but they never include both systems in the same model at the same time. 
change of average value of a behavioural trait (represented as a phenotypic trait, $p$ ) as follows:

$$
\Delta \bar{p}=\frac{1}{\bar{w}} \operatorname{cov}\left(w_{i}, g_{i}\right)+\frac{1}{\bar{w}} \operatorname{cov}\left(s_{i}, c_{i}\right)+\left\langle\Delta g_{j}\right\rangle+\left\langle\Delta c_{j}\right\rangle
$$

where the first two terms in the right-hand side measure the covariance between reproductive fitness $w_{i}$ and a genotype $g_{i}$, and cultural fitness $s_{i}$ and a cultural type $c_{i}$ (respectively). The last two terms measure the transmission bias in genetic, $\Delta g_{j}$ and cultural, $\Delta c_{j}$, inheritance. $^{12}$

After having introduced both El Mouden et al.'s and Aguilar and Akçay's versions of the Price equation, let us now check if they are formally adequate to represent cultural change. It is pretty straightforward to locate in the equations most of the different cultural forces discussed in section 2. El Mouden and collaborators explicitly locate cultural selection in the covariance term. Analogously, Aguilar and Akçay locate selection (both due to content and context-based biases) and drift in the covariance terms (El Mouden et al. do not mention drift in their article). This is not surprising since, as mentioned in section 3, also in the contemporary applications of the Price equation to non-cultural (both genetic and phenotypic) evolution, selection and drift are typically located in the covariance term (Rice, 2004; Okasha, 2006; Engen and Saether, 2014).

Both selective and random forces acting upon a population produce a nonzero covariance between cultural traits and their contribution to the next generation. While selective forces act systematically changing the frequency of cultural variants when these traits causally determine their (genetic or cultural) fitness values, random forces produce stochastic fluctuations in the frequency of cultural variants when these traits do not causally determine their fitness values. Any process based on the relationship between an arbitrary cultural trait and its contribution (i.e., the number of descendants of an individual) to the next generation is measured by the covariance term in the Price equation. This is why both selective and random forces can be put together under the label of "sorting forces".

Transmission biases affect the fidelity of transmission of cultural variants. El Mouden et al. and Aguilar and Akçay explicitly locate mutation, recombination and "individual trials" (i.e., guided variation) in the transmission term, which captures any process producing a bias in the transmission of a trait from parents to offspring.

Finally, migration can be considered an ambiguous case. None of the two cultural Price equations presented above deals explicitly with migration. Remember that the standard Price equation is derived by adopting some assumptions, some more basic than others, about the properties of an evolutionary system (see section 3 ). This equation

12 In their derivation, Aguilar and Akçay assume that the effects of genetic and cultural inheritance are additive. That is, $p=c+g+e$, where $e$ represents any non-inheritable effect due to the environment. Nevertheless, they also show how to obtain a general, non-additive, version (see Aguilar and Akçay, 2018, Appendix A5). A relevant precursor of Aguilar and Akçay's approach can be found in Frank (1998, chap. 2). He points out, regarding the Price equation, that we can use any predictors for a character $\mathrm{z}$ (e.g., alleles, group characteristics, environmental variables, but also cultural beliefs, among others). In other words, anything that is transmitted can count as a predictor. 
maps the connection between ancestors and descendants, and assumes that all descendants have the same number of ancestors. But, if organisms enter into a descendant generation from outside the population (i.e., by migration), then these organisms are not connected to any organism of the previous generation. In other words, they just appear. Therefore, it would seem that the standard Price equation cannot account for the introduction of individuals or cultural traits from outside the modelled population (i.e., we have a closed population). ${ }^{13}$ Some authors have handled this problem by adding an additional term to the standard Price equation that measures the degree of connectiveness between ancestral and descendant entities (Kerr and Godfrey-Smith, 2009). This new term would allow to represent, in the descendant population, unconnected members that arrived via migration. ${ }^{14}$

It is interesting to notice, in this regard, that both El Mouden et al. and Aguilar and Akçay's formalisms already represent different degrees of connectivity between ancestors and descendants through the covariance term. The rationale for this approach is that, in cultural evolution, the connections between ancestors and descendant populations depend on the characteristic features of social learning. Since different traits may have different cultural fitness, some ancestors can culturally influence the descendant individuals in different degrees. Moreover, descendants may differ in their number of ancestors. Both circumstances involve variation in connectivity through generations, and this phenomenon is captured by the covariance term. Thus, insofar as also migration involves variation in the number of ancestors in a population (in this case, zero ancestors for immigrated individuals), it could be properly represented in the covariance term. In such a case, the extra term proposed by Kerr and Godfrey-Smith would not be needed (see El Mouden et al., 2014, Supporting Information, p. 2).

To be precise, both cultural Price equations discussed above explicitly assume that all individuals have at least one ancestor (see El Mouden et al., 2014, Supplementing information, p. 1; Aguilar and Akçay, 2018, p. 314). This, by definition, implies that they are modelling a closed population. In other words, they are not actually dealing with migration. However, our point here is just that, in principle, both El Mouden et al. and Aguilar and Akçay's formalisms may handle migration via some simple technical modification in the

${ }_{13}$ Actually, the standard Price equation can be used to model a population with migration, but then migration (or dispersal) must be taking place between subpopulations, that are all covered by the Price equation (Frank, 1986). That is, the population is closed, but subdivided. Thus, migration can be interpreted in two ways: migration between subpopulations within the modelled population (which can be modelled with the standard Price equation), or migration in or out of the modelled population (which requires further assumptions). The classical Price equation does not cover migration in the second way, but it can in the first way (by thinking the modelled population as a meta-population, in which there is migration between subpopulations).

${ }^{14}$ It is also worthy to notice that some transmission biases may create new ideas and behaviours. In doing so, these forces are not modifying variation already present in a population but introducing new variation. Therefore, technically, they are not affecting the fidelity of transmission between ancestors and descendants, but creating new cultural traits that appears without any connection to any ancestor. Thus, the forces that provide new variation would be captured by the additional term developed by Kerr and Godfrey-Smith (2009). 
derivation to allow this kind of variation in connectivity. ${ }^{15}$ Be that as it may, researchers always have at their disposal the extra term developed by Kerr and Godfrey-Smith in order to handle migration and other processes that affect connectivity and are not captured by the weights $\Upsilon i j$.

It follows from our discussion that all the forces of cultural evolution may be, in principle, suitably located in the Price equation and, thus, that the Price equation is an adequate representation of the cultural evolutionary dynamics as characterised by dual-inheritance theory. Aguilar and Akçay's formalism is more general and, for this reason, we shall mainly refer to it in the next sections. Until now, we focused on the formal suitability of the Price equation to depict cultural change; starting from the next section, we shall assess its empirical adequacy and explanatory import.

\section{The Price equation and the causal characterisation of cultural evolutionary processes}

As mentioned in the introduction, even accepting that the conceptual framework of cultural evolutionary theory is properly summarised by the Price equation -or, more precisely, by some "properly cultural" version of the Price equation, like Aguilar and Akçay's equation - it may be questioned whether this move provides some explanatory or predictive gain. Given the highly abstract nature of the Price equation and the fact that it apparently just relates covariances, expectations and regressions, it may be argued that the equation is uncapable to provide us with any causal knowledge about the phenomena for which it supposedly accounts for. These concerns have led some researchers to consider the Price equation just as a priori truth, a "mathematical tautology" (van Veelen, 2005; Nowak and Highfield, 2011). If it were indeed the case, the Price equation would not be able to play any explanatory or predictive role. In fact, tautologies do not explain anything, they are true by definition. In addition, the critics argued that the Price equation by itself lacks of dynamic sufficiency to predict the state of the system at any time in the future beyond one generation. Frank (2012) and Luque (2017) have extensively responded to these concerns. In this context, we shall consider, more specifically, their impact on our picture of cultural evolutionary theory.

It is certainly true that the Price equation - taken as it is - has little empirical significance. If we interpret it (as we did in the last section) as a "summary" of the effects of cultural evolutionary theory, it is just a consequence law (Sober, 1984). A consequence law has a certain predictive role, insofar as it relates certain magnitudes to a specific outcome -the Price equation states that, if the two terms on the right-hand side have certain values, then the frequency of the trait under study will change correspondingly. However, consequence laws do not say anything about the causes of such an outcome and, thus, they are arguably not sufficient to provide an explanation of the phenomena under study.

This conception about the limitations of consequence laws - and, more specifically, of the Price equation - is grounded on the presupposition that, when we assess the value of a

15 One initial possibility, suggested by Erol Akçay (personal communication), could be to assume that the migrant had a hypothetical parent in the ancestral generation that had the mean parental phenotype value. 
law within a theory, we must evaluate it separately from any other part of the theory. This approach has been repeatedly questioned (see, for instance, Moulines, 1984; Sober, 1988; Díez and Lorenzano, 2013; Luque, 2017). The role of consequence laws -or, more precisely, of those consequence laws that are assumed to be fundamental - within a theory can be appreciated only if we consider the relation between the consequence laws and other elements (laws, models) of the theory. ${ }^{16}$

By assuming this perspective, we shall argue, respectively in this and the next section, that:

a) the Price equation is explanatory insofar as, when considered in relation with specific cultural evolutionary scenarios, it provides a heuristic guide about the possible causes of the phenomena under study; and

b) it is precisely because this that the Price equation (or, better, a specific cultural version of the Price equation, like Aguilar and Akçay's equation) is a good candidate to be the fundamental law, or the guiding-principle (Moulines, 1984; Díez and Lorenzano, 2013), of cultural evolutionary theory.

Not all the philosophers who have discussed the role of the Price equation within evolutionary theory have been dismissive about the possibility to derive causal considerations from it. Okasha (2006), for instance, offers solutions to various problems related to the levels of selection by providing plausible causal interpretations of the Price equation. Furthermore, Otsuka (2016) provides a causal interpretation of the Price equation with the help of causal models, and argues that this is able to settle the long-standing dispute over the causal or purely statistical character of evolutionary theory.

In order to clarify what kind of causal information can be extracted by the Price equation along with a certain description of a cultural evolutionary scenario, we shall start discussing the model built by MacCallum, Mauch, Burt and Leroi (MacCallum et al., 2012), whom explicitly employ the Price equation to explain the causes of the evolution of music (they adopt the original form of the equation, since they do not take into account genetic factors). From this model, we shall derive some general principles for the successful explanatory application of the Price equation to cultural evolution.

MacCallum et al. (2012) model music evolution through a simulation, the DarwinTunes, which works roughly as follows. The DarwinTunes stores a population of digital ge-

${ }^{16}$ Some readers may feel uncomfortable with the characterisation of the Price equation as a law. As a matter of fact, this is more commonly interpreted as a mathematical theorem concerning the relation between the aggregate properties of two sets. We think that the two characterisations need not to be at odds. According to the structuralist perspective that we adopt in section 6, laws should not be conceived as universal unrestricted empirical generalisations, like in more traditional accounts of scientific theories. In particular, all fundamental laws - like Newton's second law in Newtonian mechanicsjust denote, if taken alone, set-theoretic structures, like the Price equation. What makes these laws empirical is the fact that practitioners assume that portions of the world are isomorphic or, more vaguely, similar to those set-theoretic structures. They thus employ these laws as guiding principles to discover more restricted regularities (which, as we shall see in section 6, are the specialisations of the theory). One of our goals in this paper is precisely to show how cultural evolutionary theory can be profitably developed by assuming that the Price equation (or some version of it) is its fundamental law, in the sense we have just sketched out. 
nomes, which execute looping polyphonic sound sequences. After a certain period of time, loops may replicate to produce new loops. This process is the effect of two cultural evolutionary forces: recombination -i.e., new loops are the product of the random combination of two parents' genome - and mutation -i.e., new loops may contain novel rhythmic or melodic features. A selective pressure is then introduced via a Web interface, through which a population of consumers listen and rate samples of the loops. The consumers' ratings act as a process of cultural selection due to the content and determine the (actual) fitness of the loops; the worst rated loops are retired from the population before a new cycle of recombination occurs.

In one of the experiments with the DarwinTunes, MacCallum and his colleagues used costumers' ratings to estimate the mean fitness of the loops' population, which they denoted as $M$, the mean absolute musical appeal. The researchers observed that $M$ incremented quickly for the first 500-600 generations undergoing selection, but then apparently reached an equilibrium and started fluctuating around a mean. How might this phenomenon be explained? Since various different forces were acting on the loops' population (besides recombination, mutation and selection due to content, loops also underwent drift, due to stochastic sampling during replication), it was not obvious at all which of them was responsible for the evolutionary stasis. It is at this point that the Price equation entered the scene.

The Price equation tells us that, when a population is at evolutionary equilibrium, then $\bar{w} \Delta \bar{z}=0$. This is the case if both the covariance and the transmission terms are equal to zero, but also - and more interestingly - if the two terms are equal in magnitude but opposite in sign. Since we know that there were many evolutionary forces acting in the DarwinTunes before the population reached the equilibrium, we are quite justified in believing that we are in the second scenario. Through another experiment, MacCallum and his colleagues ascertained that the increment of $M$ over the first 500-600 generations was due to cultural selection. This entails that, during this phase, cultural selection overcame any possible hidden transmission bias contrasting it. In its turn, this reduces to two the possible hypotheses about the subsequent stasis. The first hypothesis is that cultural selective pressure suddenly decreased; the second one is that contrasting transmission biases emerged or acquired strength. ${ }^{17}$

MacCallum and his colleagues found out - by decomposing $M$ in two further values, $C_{L}$ and $R$, respectively estimating the clarity of the chordal and rhythmic structures, and consequently plotting the adaptive landscape of the DarwinTunes- that cultural selection plausibly maintains its strength during stasis. This crucially led the researchers to in-

17 To be precise, the contrasting force may be another selective pressure. To consider this possibility would complicate the discussion of the present case study, and we shall not take it into account. It is interesting to notice, nonetheless, that a similar complication can arise in classical mechanics. The application of Newton's second law to a specific scenario is compatible with an infinite number of causal decompositions. To account for a certain net force, we can postulate two forces interacting, but also 4 forces interacting with half the strength, or 8 forces with a quarter of the strength, and so on. While this is certainly a theoretical possibility, in most practical cases we can exclude most of the alternative descriptions, thanks to background knowledge or empirical tests. This plausibly applies to cultural evolutionary processes as well. At any rate, we briefly discuss how the Price equation can model scenarios in which different selective pressures interact at the end of this section. 
vestigate the presence of transmission biases opposing cultural selection. By drawing upon an analogy with genetic evolution, MacCallum and colleagues noted that "recombination could have increasingly deleterious effects if, as the population evolves, high fitness comes to depend on particular genomic configurations that can be broken up by sex; in other words, fitness epistasis increases" (2012, p. 12085). As there was evidence of synergistic fitness epistasis between $C_{L}$ and $R$, the authors thus argued that, when the population reaches a certain adaptive peak, new adaptive combinations are easily broken up by recombination (and mutation). The consequence of this is that, as observed, the adaptive evolution suddenly stops.

We have described at some length the work of MacCallum and his collaborators because we think that it is extremely didactic and illustrative of the explanatory and predictive power of the Price equation. Of course, as we have conceded to its critics, the Price equation alone is unable to detect the causes of the evolutionary change. Nonetheless, when previous knowledge about some of the forces already acting in the scenario under study is available, the Price equation provides us an invaluable heuristic guide to discover other causes of the evolutionary change (for a similar point, see Frank, 2016, pp. 13-14, and Gong et al., 2012, p. 2).

This is not just the case when $\bar{w} \Delta \bar{z}=0$ due to an interaction between selection and transmission biases, as in the DarwinTunes' equilibrium, but can be extended to a great variety of scenarios. All what is needed - besides, of course, a certain factual knowledge about the specific phenomenon being studied - is a proper formal decomposition of the Price equation. Let us clarify this point with another example. ${ }^{18}$ In sections 3 and 4 , we have seen that the covariance term of the Price equation is intended to represent both selection and drift. Nonetheless, the two evolutionary processes are not explicitly distinguished in the original formulation of the equation. In order to be able to measure the magnitude of each force, we have to separate the corresponding term in as many terms as we need to account for the phenomenon under study. Thus, for instance, Okasha (2006) suggests the following decomposition, splitting up the realised fitness $w_{i}$ in two parts -i.e., the expected fitness $w_{i}^{*}$ and its deviation $\delta_{i}-$ of the covariance term:

$$
\bar{w} \Delta \bar{z}=\operatorname{Cov}\left(w^{*}, z^{\prime}\right)+\operatorname{Cov}\left(\delta_{i}, z^{\prime}\right)
$$

representing the change due to selection and drift, respectively. In practice, this equation can be employed, analogously to the original Price equation in MacCallum et al.'s model, to explain (and possibly predict) changes in observed dynamics. If, for instance, we observe that in a certain population in equilibrium $\bar{w} \Delta \bar{z}=0$ there are not transmission biases, we know from the modified Price equation that, to understand the causes of the phenomenon, we have to look for factors reducing selective pressures or, alternatively, factors increasing the stochastic deviation from the expectation.

This explanatory strategy can be further generalised to dynamics that are not in equilibrium (that is, in which it is not the case that $\bar{w} \Delta \bar{z}=0$; Rankin et al., 2015). This can be

${ }^{18}$ See also Coulson and Tuljapurkar (2008) and Ozgul et al. (2009) for more formal decompositions of the Price equation (in this case, in evolutionary ecology). Both studies inform researchers about causal mechanisms of population change and have been applied to study actual populations (of red deer and Soay sheep, respectively). 
appreciated by focusing on the derivation of the Price equation suggested by Aguilar and Akçay (we repeat it here for the sake of clarity):

$$
\Delta \bar{p}=\frac{1}{\bar{w}} \operatorname{cov}\left(w_{i}, g_{i}\right)+\frac{1}{\bar{w}} \operatorname{cov}\left(s_{i}, c_{i}\right)+\left\langle\Delta g_{j}\right\rangle+\left\langle\Delta c_{j}\right\rangle
$$

Here, the covariance terms represent the effects of selection in genetic and cultural evolution (respectively), and the last two terms represent the transmission bias in both types of evolution. As we have seen, in cultural evolution, there are four types of selective forces possibly acting on a population: natural selection, cultural selection due to content and cultural selection due to context-based biases (frequency-dependent selection and selection due to prestige or model). Besides being an elegant reformulation of the Price equation aimed to distinguish the effects of natural selection, cultural selection due to content and other transmission biases, Aguilar and Akçay's equation can be used to convey useful information about the causes of cultural dynamics. Aguilar and Akçay themselves work out the details of some of such applications. Take, as illustration, the case in which $\Delta \bar{p}>0$, but cov$\left(w_{i}, g_{i}\right)$ and $\operatorname{cov}\left(s_{i}, c_{i}\right)$ have different signs. This is an interesting case, in which a trait spread in spite of the fact that it is either genetically or culturally unfit. ${ }^{19}$ Aguilar and Akçay show that, in a scenario in which, for instance, a high value of $p$ reduces genetic fitness (assuming the genetic transmission term is zero), the following inequality must hold true:

$$
\operatorname{cov}\left(s_{i}, c_{i}\right)>-\left(\operatorname{cov}\left(w_{i}, g_{i}\right)+\bar{w}\left\langle\Delta c_{j}\right\rangle\right)
$$

While this inequality is, in general, true regardless whether $p$ reduces or increases genetic fitness (e.g., one may deduce that $\operatorname{cov}\left(w_{i}, g_{i}\right)+\operatorname{cov}\left(s_{i}, c_{i}\right)>-\bar{w}\left\langle\Delta c_{j}\right\rangle$, if assumes that $\operatorname{cov}\left(w_{i}, g_{i}\right)$ is positive), Aguilar and Akçay are not just making a mathematical claim. Rather, they aim to provide a tool for detecting when genetically maladaptive evolution is acting in a population. The important point, as far as we can see, is that, by stating such an inequality, Aguilar and Akçay make explicit a relation between different components of fitness in a way that, if certain conditions are observed (i.e., the diffusion of a trait in spite of its poor genetic fitness), then certain causal hypotheses must be taken into account (e.g., that the diffusion of the trait is due to characteristics that made it culturally attractive). Once again, the Price equation reveals its explanatory import in the sense that it offers clues about what kind of process we should expect to find acting in a specific scenario, given certain background information about the phenomenon under study.

A last example, this time concerning the action of selection due to context-based biases, can be drawn from Gong et al. (2012). These authors employ the original Price equation to understand the evolution of languages. They are explicit about the fact they use the Price equation as an "empirical tool" (Gong et al., 2012, p. 2), that is, as an instrument to detect the influence of selective pressures on the diffusion of linguistic variants. Unbiased

19 One of the phenomena most extensively studied by cultural evolutionists, that is, the demographic transition in modern Western societies, is precisely a case in which a trait -i.e., the preference for a reduced family - spreads in spite of being genetically maladaptive. 
transmission is computationally modelled by Gong and his collaborators using a Pólya-urn model, which simulates a random diffusion of linguistic variants in a socially structured population of individuals. This null model is then complexified in a variety of ways. In most scenarios, variants are weighted by a factor corresponding to the attractiveness of the variant (which is responsible for what we called selection due to content biases). However, in some scenarios, the distribution of linguistic variants is also conditioned by the presence of individuals with a greater capacity to influence their neighbours. This factor can be, quite straightforwardly, interpreted as corresponding to a selective pressure due to prestige (or model). ${ }^{20}$

Gong et al. calculate the relative strength of selection due to prestige by running two different simulations and comparing them. In the first one, the linguistic variants are not subject to selection due to content (they are unweighted by the factor denoting the attractiveness of the variant). In this scenario, the covariance term of the Price equation is thus measuring only selection due to prestige and drift (the latter is already included in the null model). In the second scenario, both kinds of selection are acting. The notable result is that, while in the first scenario selection due to prestige fails to overcome the inertial fluctuations in the distributions of linguistic variants (i.e., the simulation is similar to the one obtained when the null model is run), in the second scenario it accelerates or delays the effects of selection due to content (depending on whether the influential individuals adopt or reject the most attractive variants). This supports the claim that selection due to prestige is indeed a specific kind of cultural selection, which —although, perhaps, not as effective as selection due to content - is able to affect the distributions of cultural variants depending on the social structure of the population.

\section{The Price equation and the structure of cultural evolutionary theory}

In the last section, we have insisted on the heuristic role that the Price equation plays when cultural evolutionists aim to identify the causes of a specific cultural dynamic. In this section, we shall further support this idea by showing how our previous characterisation of the forces of cultural evolution can be integrated within a comprehensive framework. This framework, which — in our intentions - should ideally define the structure of cultural evolutionary theory, has the Price equation, in its cultural version, at its core. In other words, we believe that the Price equation is, to all effects, the fundamental law of cultural evolutionary theory and, in this section, we shall support this claim by spelling out the unificatory role that this equation plays within the theory. ${ }^{21}$

${ }^{20}$ Notice that Gong et al.'s terminology is significatively different from the one we adopt in this article, and therefore potentially misleading. They, in fact, refer to "prestige variants" in order to denote those variants which are weighted by a selective factor that we consider, according to our framework, due to content; while they characterise the selective pressures due to prestige simply as "individual influence". Once this terminological divergence is clarified, we find that the underlying conceptions are fully compatible.

${ }^{21}$ In similar fashion, Birch (2017) has stressed the unificatory role of a particular form of the Price equation, i.e., Hamilton's rule. In Birch's view, Hamilton's rule plays the role of an organising framework in social evolutionary theory, providing heuristic guide for social evolutionary researchers. 
In order to attain this goal, we will borrow some conceptual tools from metatheoretical structuralism (Balzer et al., 1987), which is a sophisticated semantic approach to the reconstruction of the structure of scientific theories. First of all, thus, we shall introduce some terminology.

According to metatheoretical structuralism, mature scientific theories are typically composed of a set of theoretical elements arranged in an inverted-tree hierarchy, also called theory net. The theoretical elements are set-theoretic structures identifying the models of the theory. The notion of model at stake in metatheoretical structuralism is not the intuitive one that we have employed so far, but a technical one derived from model theory. In short, we can conceive of the models of the theory as those portions of the world that behave according to what is stated by the theory. At the top of the hierarchy we usually find a single theoretical element, including some very general assertion about the way in which the theory account for a class of phenomena: this is the fundamental law. The theoretical elements that branch beneath the top theoretical element are the specialisations of the top element: they specify regularities concerning some subclass of the phenomena accounted for by the theory.

In the case of Newtonian mechanics - to take a classic example- the top theoretical element is Newton's second law. Individually taken, this law is "almost (empirically) vacuous", in the sense that it does little more than providing a definition for the notion of force (Díez and Lorenzano, 2013). The domain of application of this law is specified by the other theoretical elements of the theory - such as the Newton's third law, the law of gravitation, Hooke's law, etc. - which are its specialisations. This is a possible reconstruction of Newtonian mechanics' overall structure:

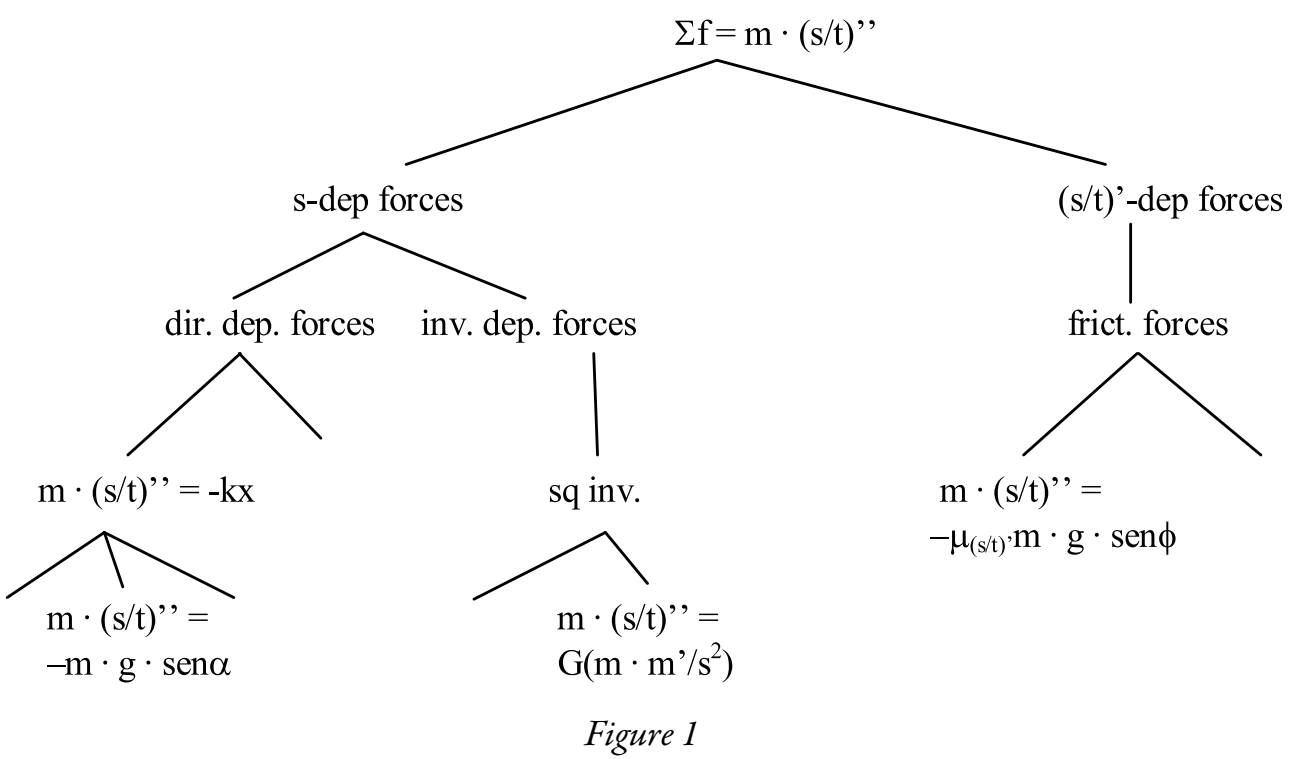

Structuralist reconstruction of Newtonian mechanics (from Díez and Lorenzano, 2013, p. 1161). The specialisations of Newton's second law are hierarchically represented as follows: the first branching distinguishes between space-dependent and velocity dependent forces; the space-dependent forces branch into direct and indirect space-dependent forces; direct space-dependent forces branch into linear negative space-dependent forces and others; and so forth 
In spite of its almost vacuous character, Newton's second law plays nonetheless an invaluable role in Newtonian mechanics: it is its guiding principle (Moulines, 1984). The guiding principle of a theory establishes "what the theory is about" by providing a very general and comprehensive schema for explaining a class of phenomena. As already suggestively noticed by Kuhn (1970), a guiding principle (which Kuhn called "generalisation-sketch") is a promise, rather than a full-fledged explanation of any phenomenon. Newton's second law gained its strength from the fact that it inspired generations of physicists to "fill the details" of the theory, by pointing out "where they had to look" in order to explain the behaviour of the physical world.

Of course, cultural evolutionary theory is not even remotely as "mature" as Newtonian mechanics. Thus, we should not expect to find in the former a well-established hierarchy of theoretical elements like in the latter. Nonetheless, we think that the Price equation provides to cultural evolutionary theory a promise of unification. The taxonomy of forces discussed in section 2 can be easily integrated into a theory net, analogous to that of Newtonian mechanics, so as to represent the structure of cultural evolutionary theory.

As already suggested, the Price equation is analogous to Newton's second law to the extent that it provides a very abstract and general statement about the kind of factors that can produce changes in the system under study (in this case, frequencies of cultural variants through time).

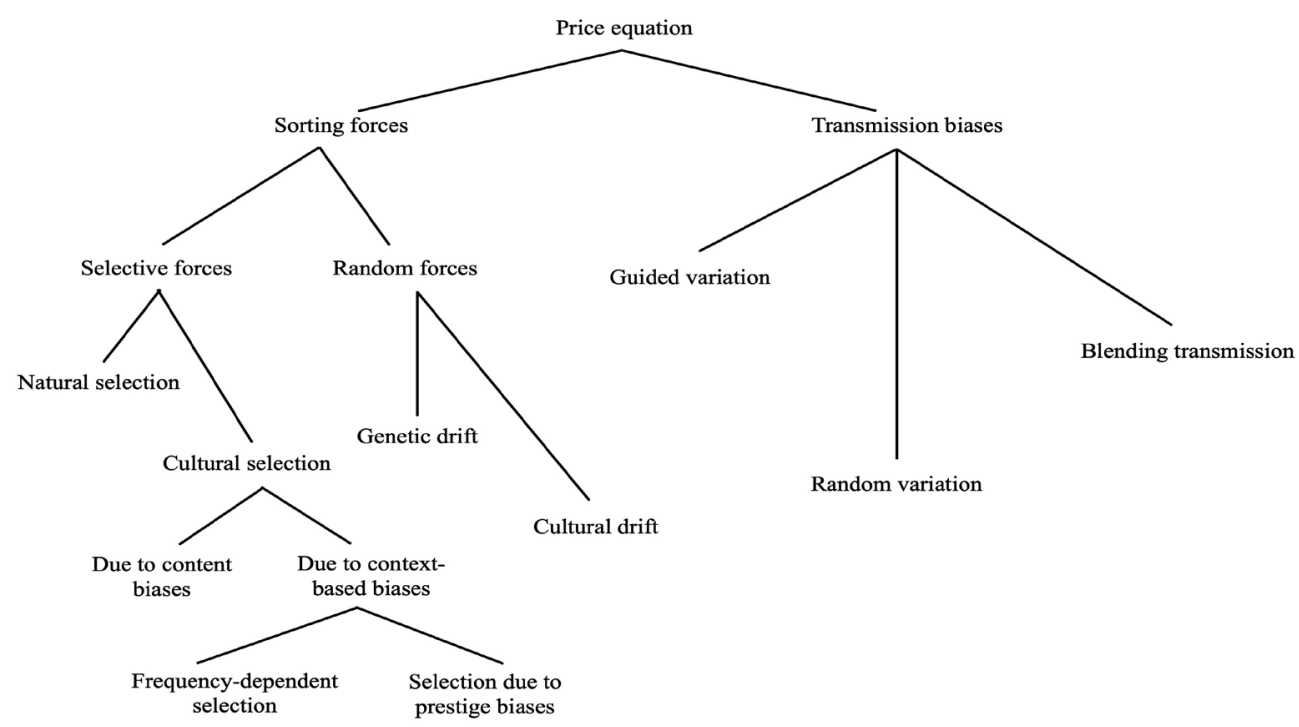

Figure 2

Tentative reconstruction of cultural dynamics theory net. It can be noticed that we omitted migration. Of course, in accordance with our discussion in section 4, migration may be located in different branches, depending on the adopted approach. If El Mouden et al.'s and Aguilar and Akçay's approach is chosen, migration may be considered as a specialisation of sorting forces. If the meta-population approach is adopted (see footnote 13), then migration is a transmission bias. Finally, another possibility would be that of adding a third major branch (Kerr and Godfrey-Smith's approach), descending directly from the Price equation, identifying what we may call "Introduction Forces" 
The relation between the Price equation -or, more properly, the cultural version of the Price equation formulated by Aguilar and Akçay - and the other nodes of the theory net should be considered as follows. The Price equation offers a mathematical partition between two possible kinds of evolutionary change: the change produced by selection, or other sorting forces (such as drift), which modify the distribution of a trait; and the change produced by transmission biases, which modify the trait itself (by modifying some of its characteristic features). This is to say that all the forces of cultural evolution can be formulated either in terms of selection or transmission biases (and migration can be incorporated, as we have seen, in different ways depending on our theoretical or empirical goals; see also Figure 2 legend). If we assume that the more appropriate formulation of the Price equation for cultural evolutionary theory is Aguilar and Akçay's equation, we may, more specifically, say that all cultural evolutionary forces are selective processes or transmission biases acting on genetic and cultural variants and their frequencies. There is no evolutionary change without these forces acting on a population of traits and, at the same time, there are no other forces acting in the evolutionary process besides them. We may say that the cultural Price equation contain all the fundamental concepts of the theory.

While the Price equation describes the net change in the diffusion of a trait as the product, or effect, of forces (remember that it is a consequence law and, thus, it is not -if taken alone- causally meaningful), the specialisations of the theory provide overall causal characterisations of each force, and these characterisations allow, in their turn, empirical descriptions of the processes acting in cultural change. ${ }^{22}$ Of course, what concretely counts as a specific sorting factor or transmission bias in a real-world scenario is different from what counts as a sorting factor or transmission bias in another scenario. This is analogous to what occurs in "natural" populations, where the way in which natural selection or other evolutionary factors act is strongly dependent on the ecological circumstances. Nevertheless, a precise characterisation of the overall causal features of the evolutionary factors is what enables, in line with what we have seen in section 5, reliable hypotheses about concrete scenarios.

Although in most specialisations of the theory one of the terms of the cultural Price equation is equal to zero (this allows to put in the foreground the characteristic features of the single force accounted for in each specialisation), the Price equation applies in all of them. The equation has such a general range of application precisely thanks to its "empirical emptiness". This, as argued throughout the last two sections, is not a defect, but rather a major strength of the Price equation. Thanks to its "almost a priori" and constitutive character, it determines an ideal explanatory pattern (Kitcher, 1989) for the specialisations of the theory. In this way, it manages to play a systematising role with respect to the structure of the theory and to coordinate modellers' effort to provide coherent explanations of the phenomena of cultural change.

${ }^{22}$ In the literature there are already good candidates for the mathematical representation of some of the specialisations of the theory. We have already mentioned some of them in the previous sections of this article. We have chosen not to include them in Figure 2 because we think that, albeit valuable, they have not been systematically conceived within a general framework like the one we are presenting here and, thus, they are not fully consistent to each other. 


\section{Concluding remarks}

The Price equation is nowadays used in many areas of biology to represent the processes of population change. In spite of - or, we may say, thanks to- its highly abstract way to depict the population change, the Price equation provides a powerful conceptual resource. Instead of univocally identify the causes of population change (like most equations in population genetics), it classifies their common effects in two simple terms. Rather than being directly applicable to model specific scenarios, it works as a versatile heuristic tool to formulate new hypotheses about the phenomena under study, as well as to find out new equations describing these more specific scenarios. The Price equation can be considered the fundamental law of evolution (Luque, 2017; Queller, 2017; Luque and Baravalle, 2021) insofar as it does not commit us to any unequivocal view about the causes of evolution, but it identifies those concepts and dynamical features that any evolutionary approach to a certain domain of phenomena must assume.

Cultural evolutionary theory is an expanding area of research. While it is broadly intended - as its name itself states - as an evolutionary theory and cultural evolutionary theorists commonly employ formal tools derived from population genetics, little effort has been paid to define the underlying principles connecting cultural evolutionary models. In this article, we have argued that cultural evolutionary theory is an evolutionary theory because the forces of cultural evolution (as usually conceptualised in dual-inheritance theory) can be easily plugged in a theoretical structure in which a specific cultural version of the Price equation is the fundamental law. As well as in other domains of evolutionary theory, the Price equation works in cultural evolutionary theory as a heuristic principle, providing a guide to the formulation of explanations of specific phenomena (as we have seen in section 5) and a unifying framework to think cultural evolutionary processes collectively (as we have argued in section 6).

Our aim with this work has been to suggest that, in spite of the fact that cultural evolutionary theory is frequently presented as a quite heterogenous set of models, there are no reason to be too pessimistic about its theoretical unity. The Price equation provides the key to integrate different factors producing cultural change and accumulation in a coherent picture. Albeit the details of our metatheoretical reconstruction are purely tentative, and may be certainly improved, we are convinced that the future developments of cultural evolutionary theory will confirm the foundational and systematising role that the Price equation plays in it.

\section{Acknowledgements}

LB would like to thank the Brazilian National Council for Scientific and Technological Development (CNPq Grant No402619/2016-1) and the Fundação para a Ciência e a Tecnologia de Portugal (FCT Contract No DL57/2016/CP1479/CT0064) for the financial support. VL was supported by the Spanish Ministry of Science, Innovation and Universities under Juan de la Cierva fellowship (FJC2018-038661-I), and Projects FFI2016-76799-P and FFI2017-89639-P. Special thanks to Jonathan Birch and one anonymous reviewer for their useful comments, and to Erol Akçay and Daniel Nettle for helpful discussions and clarifications on their respective works. 


\section{REFERENCES}

Acerbi, A. \& Mesoudi, A. (2015). If we are all cultural Darwinians what's the fuss about? Clarifying recent disagreements in the field of cultural evolution. Biology \& Philosophy, 30, 481-503.

Aguilar, E. \& Akçay, E. (2018). Gene-culture co-inheritance of a behavioral trait. The American Naturalist, 23, 311-320.

Alizon, S. (2009). The Price equation framework to study disease within-host evolution. Journal of Evolutionary Biology, 22, 1123-1132.

Balzer, W., Moulines, C. U., \& Sneed, J. D. (1987). An architectonic for science. The structuralist program. Dordrecht: Reidel.

Baravalle, L. (2021). Cultural evolutionary theory as a theory of forces. Synthese, 198, 2801-2820.

Birch, J. (2017). The philosophy of social evolution. Oxford: Oxford University Press.

Boyd, R. \& Richerson, P. J. (1985). Culture and the evolutionary process. Chicago: The University of Chicago Press.

Brandon, R. (1982). A structural description of evolutionary theory. In P. D. Asquith \& R. N. Giere (Eds.), PSA 1980 (Vol. II, pp. 427-439). East Lansing, Michigan: Philosophy of Science Association.

Cavalli-Sforza, L. L. \& Feldman, M. (1981). Cultural transmission and evolution. Princeton: Princeton University Press.

Charbonneau, M. (2020). Understanding cultural fidelity. British Journal for the Philosophy of Science, 71, 1209-33.

Charbonneau, M. \& Bourrat, P. (2021). Fidelity and the grain problem in cultural evolution. Synthese. doi:10.1007/s11229-021-03047-1.

Claidière, N., Scott-Phillips, T. C., \& Sperber, D. (2014). How darwinian is cultural evolution? Philosophical Transactions of the Royal Society $B, 369$.

Coulson, T. \& Tuljapurkar, S., (2008). The dynamics of a quantitative trait in an age-structured population living in a variable environment. The American Naturalist, 172(5), 599-612.

Díez, J. \& Lorenzano, P. (2013). Who got what wrong? Fodor and Piattelli on Darwin: Guiding principles and explanatory models in natural selection. Erkenntnis, 78, 1143-1175.

El Mouden, C., André, J.-B., Morin, O., \& Nettle, D. (2014). Cultural transmission and the evolution of human behaviour: A general approach based on the Price equation. Journal of Evolutionary Biology, 27, 231-241.

Engen, S. \& Saether, B. E. (2014). Evolution in fluctuating environments: Decomposing selection into additive components of the Robertson-Price equation. Evolution, 68, 854-865.

Frank, S. A. (1986). Dispersal polymorphism in subdivided populations. Journal of Theoretical Biology, 122, 303-309.

Frank, S.A. (1998). Foundations of social evolution. Princeton: Princeton University Press.

Frank, S. A. (2012). Natural selection. IV. The Price equation. Journal of Evolutionary Biology, 25, 10021019.

Frank, S. A. (2016). The inductive theory of natural selection: Summary and synthesis. arXiv:1412.1285. Retrieved from: https://arxiv.org/abs/1412.1285

Frank, S. A. (2017). Universal expressions of population change by the Price equation: Natural selection, information, and maximum entropy production. Ecology and Evolution, 7, 3381-3396.

Gardner, A. (2011). Kin selection under blending inheritance. Journal of Theoretical Biology, 284, 125-9.

Godfrey-Smith, P. (2009). Darwinian populations and natural selection. Oxford: Oxford University Press.

Gong, T., Shuai, L., Tamariz, M., \& Jäger, G. (2012). Studying language change using Price equation and Pólya-urn dynamics. PLos ONE, 7, e33171. Retrieved from: https://journals.plos.org/plosone/article?id=10.1371/journal.pone. 0033171

Govaert, L., Pantel, J.H., \& De Meester, L. (2016). Eco-evolutionary partitioning metrics: assessing the importance of ecological and evolutionary contributions to population and community change. Ecology letters, 19(8), 839-853. 
Helanterä, H. \& Uller, T. (2010). The Price equation and extended Pólya-urn dynamics inheritance. Philosophy and Theory in Biology, 2, e101.

Henrich, J. (2001). Cultural transmission and the diffusion of innovations: Adoption dynamics indicate that biased cultural transmission is the predominate force in behavioural change. American Anthropologist, 103, 992-1013.

Henrich, J. (2004). Demography and cultural evolution: How adaptive cultural processes can produce maladaptive losses: The Tasmanian case. American Antiquity, 68, 197-214.

Henrich, J. \& Boyd, R. (2002). On modeling cultural evolution: Why replicators are not necessary for cultural evolution. Journal of Cognition and Culture, 2, 87-112.

Kendal, R. L., Boogert, N. J., Rendell, L., Laland K. N., Webster, M., \& Jones P. L. (2018). Social learning strategies: Bridge-building between fields. Trends in Cognitive Science, 22, P651-665.

Kerr, B. \& Godfrey-Smith, P. (2009) Generalization of the Price equation for evolutionary change. Evolution, 63, 531-536.

Kitcher, P. (1989). Explanatory unification and the causal structure of the world. In P. Kitcher, \& W. C. Salmon (Eds.), Scientific explanation (pp. 410-505). Minneapolis: University of Minnesota Press.

Kuhn, T. S. (1970). Second thoughts on paradigms. In F. Suppe (Ed.) The structure of scientific theories (pp. 459-482). Urbana: University of Illinois Press.

Lewens, T. (2015). Cultural evolution. Oxford: Oxford University Press.

Lewontin, R. (1974). The genetic basis of evolutionary change. New York: Columbia University Press.

Lumsden, C. J. \& Wilson, E. O. (2005[1981]) Genes, mind, and culture. London: World Scientific.

Luque, V. J. (2017). One equation to rule them all: A philosophical analysis of the Price equation. Biology and Philosophy, 32, 97-125.

Luque, V. J. \& Baravalle, L. (2021). The mirror of physics: On how the Price equation can unify evolutionary biology. Synthese, 199, 12439-12462.

MacCallum, R. M., Mauch M., Burt A., \& Leroi A. M. (2012) Evolution of music by public choice. PNAS, 109, 12081-12086.

Mesoudi, A. (2011). Cultural evolution: How Darwinian theory can explain human culture and synthesize the social sciences. Chicago: The University of Chicago Press.

Moulines, C. U. (1984). Existential quantifiers and guiding principles in physical theories. In J. J. E. Gracia, E. Rabossi, E. Villanueva \& M. Dascal (Eds.) Philosophical analysis in Latin America (pp. 173-198). Dordrecht: Reidel.

Nowak, M. A., \& Highfield, R. (2011). SuperCooperators: Altruism, evolution, and why we need each other to succeed. New York: Free Press.

Odling-Smee, F. J., Laland K. N., \& Feldman M. W. (2003). Niche construction: The neglected process in evolution. Princeton: Princeton University Press.

Okasha, S. (2006). Evolution and the levels of selection. New York: Oxford University Press.

Okasha, S. (2007). Cultural inheritance and Fisher's "Fundamental Theorem" of natural selection. Biological Theory, 2, 290-299.

Otsuka, J. (2016). Causal foundations of evolutionary genetics. The British Journal for the Philosophy of Science, 67, 247-269.

Ozgul, A., Tuljapurkar, S., Benton, T.G., Pemberton, J.M., Clutton-Brock, T.H., \& Coulson, T. (2009). The dynamics of phenotypic change and the shrinking sheep of St. Kilda. Science, 325(5939), 464-467.

Price, G. R. (1970). Selection and covariance. Nature, 227, 520-521.

Price, G. R. (1972). Extension of covariance selection mathematics. Annals of Human Genetics, 35, 485-490.

Price, G. R. (1995). The nature of selection. Journal of Theoretical Biology, 175(3), 389-396.

Queller, D. (2017). Fundamental theorems of evolution. American Naturalist, 189, 345-353.

Rankin, B. D., Fox, J. W., Barrón-Ortiz, C. R., Chew, A. E., Holroyd, P. A., Ludtke, J. A., Yang, X., \& Theodor, J. M. (2015). The extended Price equation quantifies species selection on mammalian body size across the Palaeocene/Eocene thermal maximum. Proceedings of the Royal Society B, 282, 20151097. 
Rice, S. H. (2004). Evolutionary theory: Mathematical and conceptual foundations. Sunderland, MA: Sinauer Associates.

Rice, S.H. (2008). A stochastic version of the Price equation reveals the interplay of deterministic and stochastic processes in evolution. BMC evolutionary biology, 8(1), 1-16.

Rice, S.H. (2020). Universal rules for the interaction of selection and transmission in evolution. Philosophical Transactions of the Royal Society B, 375(1797), 20190353.

Sober, E. (1984). The nature of selection. Cambridge, MA: MIT Press.

Sober, E. (1988). Reconstructing the past: Parsimony, evolution, and inference. Cambridge, MA: MIT Press. van Veelen, M. (2005). On the use of the Price equation. Journal of Theoretical Biology, 237, 412-426.

Walsh, B. \& Lynch, M. (2018) Evolution and selection of quantitative traits. Oxford: Oxford University Press.

Lorenzo Baravalle is currently researcher at the Faculty of Science of the University of Lisbon. His research interests are evolutionary dynamics in biology, and the relation between biology, mathematics and computation.

Address: Universidade de Lisboa, Campo Grande; Edifício C4-30 piso, Sala 4.3 .24 (Lisbon, 1749-016; Portugal). E-mail: Ibaravalle@fc.ul.pt. ORCID: https://orcid.org/0000-0001-6299-6403

Víctor J. Luque is an Assistant Professor at the Dpt. of Philosophy (University of Valencia). His work is focused on the structure of evolutionary theory, its mathematical models, and the causes of evolution.

Address: Department of Philosophy, University of Valencia. Avda. Blasco Ibáñez, 30 - Planta Setena (46010 Valencia-Spain). E-mail: victor.luque@uv.es. ORCID: https://orcid.org/0000-0002-2911-1105. 
